\title{
Creatività vs patrimonio culturale? The winner takes it all ${ }^{1}$
}

\section{Mara Cerquetti}

\begin{abstract}
Obiettivo del paper: Il presente contributo analizza le origini, le caratteristiche e gli effetti dello slittamento verso la creatività a cui si è assistito nelle politiche culturali a livello europeo nel corso degli ultimi vent'anni, al fine di comprenderne l'impatto sulla gestione del patrimonio culturale e dei musei.

Metodologia: Il lavoro verifica i risultati della ricognizione della letteratura su questo tema attraverso l'analisi del caso del Regno Unito, paese europeo in cui ha avuto origine l'approccio basato sulla creatività. Dopo aver ripercorso il dibattito scientifico sulle industrie culturali e creative (ICC), vengono messi in luce la nascita e lo sviluppo delleconomia creativa. La ricerca è basata sullesame dettagliato di articoli scientifici, documenti di policy e report e convalidata da alcune interviste in profondità ad un campione di musei londinesi.

Risultati: I risultati della ricerca rivelano come lo spostamento verso la creatività abbia concorso alla marginalizzazione del cultural heritage nelle politiche culturali in favore delle ICC stricto sensu e alla conseguente affermazione di modelli che non sempre tengono conto della specificità e del valore delle risorse locali. Limiti sono stati registrati anche nel conseguimento degli obiettivi di inclusione sociale e democratizzazione culturale e nella promozione della cross-fertilization tra i diversi settori culturali.
\end{abstract}

Limiti della ricerca: Il lavoro ha i limiti della ricerca qualitativa. Un suo ulteriore sviluppo, oltre all'ampliamento dell'oggetto dell'indagine, potrebbe comprendere l'analisi dei risultati della programmazione culturale europea.

Implicazioni pratiche: Il contributo fornisce suggerimenti a policy makers e manager pubblici e privati operanti nel settore culturale proponendo di esplorare modelli alternativi basati sulla valorizzazione delle risorse place-specific ai fini dello sviluppo sostenibile nel contesto glocale.

Originalità del paper: Nel contesto del crescente entusiasmo verso forme di sviluppo creative-driven la ricerca mette in luce alcuni limiti dei modelli predominanti.

Parole chiave: patrimonio culturale; musei; industrie culturali e creative; politiche culturali

La ricerca da cui nasce questo lavoro è stata finanziata dall'Università di Macerata nel 2015 attraverso il "BANDO GIOVANI RICERCATORI - a.a. 2014-2015”. Lautrice ringrazia l'ICCE - Institute for Creative and Cultural Entrepreneurship, Goldsmiths University of London, per l'ospitalità nel periodo da gennaio ad aprile 2016. Un ringraziamento speciale va ai musei che hanno partecipato alla ricerca, in particolare alle persone che hanno accettato di essere intervistate. 


\section{sinergie Creativity vs cultural heritage? The winner takes it all}

Purpose of the paper: The paper analyses the origins, features and effects of the shift towards creativity in cultural policy over the last twenty years, aiming at understanding its impact on cultural heritage and museum management.

Methodology: The research verifies the findings of the literature review on this matter analysing the case of the UK as the European country where the creativitybased approach was born. After going over the scientific debate on cultural and creative industries (CCI), the beginning of creative economy and its development are pointed out. The research is based on the deep examination of scientific articles, policy documents and reports and strengthened by some in-depth interviews to a sample of museum managers in London.

Results: The research results reveal that the shift towards creativity has marginalised cultural heritage in cultural policy in favour of CCI stricto sensu, determining the predominance of models that do not consider the specificity and value of local resources. Goals of social inclusion, democratization of culture and cross-fertilization between different cultural sectors too have not been fully achieved.

Research limitation: This paper has the limitations of the qualitative research. A further development could encompass the widening of the field of investigation and the analysis of the results of European cultural programmes.

Practical implications: The paper provides advice to policy makers and public and private cultural managers, suggesting the opportunity of a deeper exploration of alternative models based on the enhancement of place-specific resources and aimed at promoting sustainable development in the glocal context.

Originality of the paper: In the context of an increasing enthusiasm towards creative-driven approaches the research highlights some limits of the prevailing models.

Key words: cultural heritage; museums; cultural and creative industries; cultural policies

Se cè un continuo arricchimento di termini tratti dagli studi specializzati (processo da tempo in atto nell'italiano), quello che è acquisito dalla lingua non è il rigore lessicale ma sono solo le sue immagini sonore, non è la soddisfazione di stringere la realtà in modo che non scappi ma è un nuovo sistema di allusioni, non è la fondamentale democraticità di un rapporto tecnico con le cose ma un nuovo accento dell'Autorità.

(Calvino, 1995 [1965], p. 147)

\section{Introduzione}

"Ma come parla? [...] Chi parla male, pensa male e vive male. Bisogna trovare le parole giuste: le parole sono importanti!", gridava ad una giornalista Michele Apicella in unormai celebre scena di "Palombella rossa”, film del 1989 diretto e interpretato da Nanni Moretti. Nell'arco degli ultimi vent'anni, nelle scienze sociali il valore delle parole è stato oggetto di vari studi, che hanno preso in esame ora il potere discriminante del linguaggio (Butler, 1997) ora, e più di recente, il "cedimento linguistico" a cui si sta assistendo nell'era della finanza derivata (Appadurai, 2015). Il linguaggio viene così riconosciuto come un potente strumento per reinventare l'ordine del mondo sostituendone gli ideali di riferimento (Caust, 2003, p. 56). 
Lungo il solco di questa sensibilità alle parole, il presente contributo riflette sul linguaggio che a partire dalla fine degli anni '90 del Novecento si è progressivamente imposto nelle politiche culturali e sulla Weltanschauung che lo sottende, concentrandosi sulle implicazioni per il management culturale. In particolare il lavoro focalizza l'attenzione sul ruolo crescente della creatività nelle politiche culturali e sulla progressiva emergenza delle industrie culturali e creative (ICC), per comprenderne leffetto sulla gestione del cultural heritage e degli istituti culturali preposti alla sua conservazione e valorizzazione come i musei. Tenendo conto delle più recenti innovazioni concettuali condivise a livello internazionale, in questa sede per cultural heritage o eredità culturale ${ }^{2}$ si intende

un insieme di risorse ereditate dal passato che le popolazioni identificano, indipendentemente da chi ne detenga la proprietà, come riflesso ed espressione dei loro valori, credenze, conoscenze e tradizioni, in continua evoluzione. Essa comprende tutti gli aspetti dell'ambiente che sono il risultato dell'interazione nel corso del tempo fra le popolazioni e i luoghi (Council of Europe, 2005, art. 2).

Dopo aver analizzato i contenuti e gli obiettivi delle più recenti politiche europee in materia, la ricerca prende in esame alcuni rapporti prodotti a livello nazionale, mettendo in luce la confusione di termini e di categorie che li contraddistingue. Considerata l'influenza che certe classificazioni hanno negli investimenti, nellorientamento delle politiche culturali e nella definizione delle strategie manageriali, si ripercorre l'affermarsi della creatività prima e delle ICC poi nelle politiche culturali, con particolare riferimento al dibattito scientifico che ha accompagnato l'utilizzo e levoluzione di certi concetti nell'ambito degli studi economico-gestionali. Centrale in questo dibattito è stato il contributo degli studi anglosassoni e, in Europa, delle politiche avviate in Gran Bretagna a partire dalla seconda metà degli anni '90 del Novecento fino alla fine del primo decennio del XXI secolo. Per molti studiosi, infatti, è stato a seguito della vittoria dei New Labour nel 1997 che si è assistito ad un decisivo slittamento semantico e che il termine "industrie creative" ha conquistato la supremazia nelle politiche pubbliche (Galloway e Dunlop, 2007, p. 18). Questo orientamento viene indagato prendendo in esame articoli scientifici, rapporti di ricerca $\mathrm{e}$ documenti di policy e ulteriormente verificato attraverso alcune interviste in profondità ad un campione di musei londinesi, diversi per titolo proprietario, dimensioni e tipologia delle collezioni.

L’obiettivo è quello di comprendere se l'attuale attenzione ai settori culturali e creativi costituisca unopportunità o una minaccia per il patrimonio culturale in paesi, come l'Italia, in cui la sua capillare diffusione

2 Considerando la sua diffusione nel linguaggio comune, nel presente contributo l'espressione "patrimonio culturale" viene utilizzata nell'accezione (più ampia) dell'inglese cultural heritage (letteralmente "eredità culturale"). Stricto sensu, nel contesto giuridico italiano, il patrimonio culturale ha però una nozione più circoscritta, coincidente con l'insieme dei beni culturali e paesaggistici di cui all'art. 2 del Codice dei beni culturali e del paesaggio (D. Lgs. 22 gennaio 2004, n. 42), e non comprende dunque il patrimonio culturale immateriale incluso invece nella nozione di cultural heritage (Cammelli, 2017, pp. 16-17).
Mara Cerquetti Creatività vs patrimonio culturale? The winner takes it all 
sinergie Vol. 36, N. 105, 2018

costituisce un vantaggio competitivo inimitabile a livello internazionale. Nello specifico, da un lato, si cercherà di capire se nellattuale dibattito il cultural heritage sia stato marginalizzato e se le politiche culturali siano diventate esclusivamente le politiche delle ICC; dallaltro, se lo slittamento verso la creatività abbia promosso la cross-fertilization tra diversi settori aiutando le organizzazioni operanti nel settore del patrimonio culturale a migliorare le loro performance e a raggiungere la propria missione.

Nella parte conclusiva del lavoro si propone una possibile declinazione dell'approccio emergente che non perda di vista le risorse idiosincratiche di un territorio, ovvero i place-specific assets sui quali poter innestare strategie di sviluppo sostenibile e duraturo a livello locale, ma in ottica globale (Oakley, 2004).

\section{Culturale o creativo? Questo è il problema}

\subsection{La strategia europea per i settori culturali e creativi}

Nell'aprile del 2010, in linea con la strategia "Europa 2020" e alcune delle sue "iniziative faro", la Commissione Europea ha pubblicato il Libro Verde sulle ICC, Unlocking the potential of cultural and creative industries ("Le industrie culturali e creative, un potenziale da sfruttare"), al fine di promuovere un approccio strategico che faccia dell'"eccezionale patrimonio culturale [europeo] la base di una solida economia creativa e della coesione della società" (Commissione Europea, 2010, p. 4). In tale documento si opta per il termine "industrie" al posto di "settori", adottando al contempo una nozione ampia e sui generis di $\mathrm{ICC}^{4}$, comprensiva del patrimonio culturale e del settore pubblico.

Puntando a promuovere la cross-fertilization tra le diverse componenti del settore culturale e creativo, il Libro Verde non mette in luce, pur

3 Si vedano in particolare: "L'Unione per l'innovazione", "Un'agenda europea del digitale", "Un'agenda per nuove competenze e nuovi posti di lavoro" e "Una politica industriale per l'era della globalizzazione".

4 Secondo la definizione fornita dal Libro Verde "le 'industrie culturali' sono le industrie che producono e distribuiscono beni o servizi che, quando vengono concepiti, sono considerati possedere un carattere, un uso o uno scopo specifici che incorporano o trasmettono espressioni culturali, quale che sia il loro valore commerciale. Oltre ai settori tradizionali delle arti (arti dello spettacolo, arti visive, patrimonio culturale - compreso il settore pubblico), questi beni e servizi comprendono anche i film, i Dvd e i video, la televisione e la radio, i giochi video, i nuovi media, la musica, i libri e la stampa. Questo concetto è definito in relazione alle espressioni culturali nel contesto della convenzione UNESCO sulla protezione e la promozione della diversità delle espressioni culturali (2005). Le 'industrie creative' sono le industrie che utilizzano la cultura come input e hanno una dimensione culturale, anche se i loro output hanno un carattere principalmente funzionale. Comprendono l'architettura e il design, che integrano elementi creativi in processi più ampi, e sottosettori come il design grafico, il design di moda o la pubblicità. A un livello più periferico, molti altri settori, tra l'altro quelli del turismo e delle nuove tecnologie, si basano sulla produzione di contenuti per il loro sviluppo e sono quindi in certa misura interdipendenti con le industrie culturali e creative. Questi settori non sono compresi esplicitamente nel concetto di industrie culturali e creative utilizzato in questo Libro verde" (Commissione Europea, 2010, p. 6). 
distinguendole, le differenze tra cultural heritage e performing arts, tra industrie culturali e industrie creative. Nello specifico la strategia promossa in questo documento non considera i differenti bisogni e le caratteristiche distintive delle diverse organizzazioni operanti nel settore culturale e creativo, a partire dalle differenze tra beni pubblici, caratterizzati da non rivalità e non escludibilità dal consumo, e in grado di generare esternalità positive non remunerate all'atto dello scambio (es. patrimonio culturale), e beni privati, caratterizzati da rivalità ed escludibilità e scambiati ad un prezzo di mercato (es. media e stampa), o più in generale tra attività culturali "profittevoli" e "non profittevoli", da alcuni ritenute sostanziali per decidere le politiche settoriali più appropriate (Valentino, 2012, p. 61). Altra differenza trascurata, e connessa alle precedenti, è quella tra il patrimonio culturale, come testimonianza materiale e immateriale dell'umanità e del suo ambiente, e l'arte e le attività culturali svolte nel presente, che non necessariamente entreranno a far parte del patrimonio culturale europeo.

Lo stesso approccio è oggi condiviso da "Europa Creativa", il programma dell'Unione Europea per i settori culturali e creativi 2014-2020, finalizzato a promuovere e a salvaguardare la diversità linguistica e culturale europea e a rafforzare la competitività del settore culturale e creativo per favorire una crescita economica intelligente, sostenibile e inclusiva. Come da alcuni suggerito, ci sarebbe da capire se alla base di tale approccio indifferenziato non vi sia la volontà di dare maggior peso al settore industriale culturale e creativo stricto sensu. Allorigine potrebbero esservi "le pressioni dei paesi del centro-nord Europa (molto più presenti nel settore delle CCI [Cultural and Creative Industries] che non del patrimonio culturale rispetto ai paesi del centro-sud Europa), che in questo modo potranno, nel periodo 20142020, godere di vantaggi rilevanti nellottenere finanziamenti europei, rispetto ai paesi del centro-sud Europa nei quali prevale il cultural heritage" (Donato, 2013, p. 32).

Una risposta adeguata a tale quesito potrà essere fornita alla scadenza della programmazione 2014-2020, attualmente ancora in corso, attraverso l'analisi e la valutazione di tutti i progetti finanziati, dei relativi beneficiari, delle partnership costituite e dei risultati conseguiti. Certo è, però, che tale approccio ha contribuito ad alimentare un po' di confusione nella comprensione di un macro-settore i cui contenuti e confini sono già abbastanza ambigui e sfuggenti. Non si mette qui in dubbio lo slancio, positivo e rilevante, nei confronti degli investimenti nel settore culturale, ma non si può nemmeno trascurare il rischio di un appiattimento che può essere dannoso nella misura in cui impedisce di vedere il peso di gerarchie ed esclusioni e di individuare le reali lacune del sistema e, di conseguenza, le corrette strategie per colmarle.

\subsection{Confini di difficile definizione}

Il 22 febbraio 2014, al momento del suo insediamento, il ministro dei Beni, delle Attività Culturali e del Turismo, Dario Franceschini, ha definito il suo ministero "il più importante ministero economico italiano" (E\&Y, 2015 , p. 3). Il valore economico del patrimonio culturale, in effetti, è oggi
Mara Cerquetti culturata vs patrimonio it all 
sinergie Vol. 36, N. 105, 2018

ampiamente riconosciuto. Come stabilito dalla Convenzione di Faro, firmata dall'Italia nel 2013, ma ancora in attesa di ratifica, leredità culturale è source e resource, in grado di promuovere non solo la diversità culturale e una migliore qualità della vita, lo sviluppo umano e la costruzione di una società pacifica e democratica, ma anche la creatività contemporanea e lo sviluppo sostenibile (Council of Europe, 2005). Al fine di raggiungere questi obiettivi anche il recente rapporto Cultural heritage Counts for Europe adotta un approccio fondato su quattro pilastri, per misurare l'impatto sociale, economico, culturale e ambientale del patrimonio culturale (CHCfE, 2015).

Tuttavia, da un punto di vista economico, quando si analizzano il ruolo e l'impatto dei settori culturali, il patrimonio culturale è spesso incluso in un più ampio spettro di attività culturali e creative i cui confini sono alquanto incerti e in cui la posizione del cultural heritage non è ben definita. In Italia, secondo il primo studio sulle ICC, Italia Creativa, nel 2014 il settore culturale e creativo ha generato un valore economico complessivo pari a 40,1 miliardi di euro (E\&Y, 2015, p. 10), impiegando 850.000 persone corrispondenti al 3,8\% della forza lavoro italiana (E\&Y, 2015, p. 17 e p. 27$)^{5}$, mentre secondo il rapporto Io sono cultura nello stesso anno il Sistema Produttivo Culturale e Creativo ha prodotto 78,6 miliardi di euro, impiegando il 5,9\% della forza lavoro (Symbola e Unioncamere, 2015, p. 25). Tali discrepanze dipendono dai differenti metodi applicati nei due rapporti per disegnare il perimetro del settore culturale e creativo: mentre il primo considera 11 sub-settori culturali e creativi (architettura, arti performative, arti visive, cinema, libri, musica, pubblicità, quotidiani e periodici, radio, televisione e home entertainment, videogiochi) (E\&Y, 2015, p. 12), il secondo identifica 5 categorie nellambito delle ICC (patrimonio culturale, arti performative e visive o attività culturali non riproducibili, industrie culturali, industrie creative - design, architettura e comunicazione - e attività creative-driven, comprendenti tutte quelle attività economiche che hanno una stretta sinergia con il settore culturale e creativo) (Symbola e Unioncamere, 2015, pp. 51-52).

Analizzando nel dettaglio il ruolo del patrimonio culturale, il rapporto di Ernst \& Young include musei, monumenti e siti archeologici nella categoria "arti visive", escludendo biblioteche e archivi, che pure sono istituti preposti alla tutela e valorizzazione del patrimonio culturale. Si rinnova così la confusione tra "arte" e "patrimonio culturale" (Cerquetti, 2014; Montella, 2016), già alimentata dal rapporto Economy of Culture in Europe (KEA European Affairs, 2006), che colloca il cultural heritage nel "core arts field". Infine, se si vuole comprendere il valore del patrimonio culturale, sarà bene metterne in luce gli specifici punti di forza e di debolezza rispetto agli altri sub-settori. Quando se ne analizza il contributo al PIL e alloccupazione, il patrimonio culturale e gli altri sub-settori hanno infatti differenti performance. Nel 2015, il valore aggiunto generato dalle attività creative-driven è stato del 2,3\% e quello delle industrie culturali del $2,2 \%$, mentre il patrimonio culturale ha raggiunto solo lo 0,2\% (Symbola e Unioncamere, 2016, p. 63). Molto simile è la situazione riguardante loccupazione: $2,4 \%$ è la percentuale degli occupati nelle attività creative-

5 Tali dati si riferiscono alle attività e agli impiegati diretti. 
driven, $2 \%$ quella nelle industrie creative e solo $0,2 \%$ nel patrimonio culturale (Symbola e Unioncamere, 2016, p. 64).

I risultati non sono rassicuranti neanche quando si considera il ritorno economico generato dal patrimonio culturale: nel 2007, l'Italia ha ottenuto un ritorno medio di 0,5 milioni di euro per sito culturale, mentre, nello stesso anno, negli USA il ritorno è stato 8,16 volte superiore a quello italiano; 1,8 milioni di euro sono stati generati dalla Francia e 3,3 milioni di euro dal Regno Unito (E\&Y, 2015, p. 4). Tuttavia l'effetto moltiplicatore del patrimonio culturale è il più alto dopo quello del fiorente settore creativedriven - rispettivamente 2,09 e 2,20 nel 2015 (Symbola e Unioncamere, 2016, p. 96). Il patrimonio culturale, infatti, va considerato un bene pubblico che genera esternalità positive non solo nei settori connessi, ma anche in quelli correlati, come il turismo e l'agroalimentare.

Tracciando alcune considerazioni preliminari sul significato di tali dati, si rileva che i risultati spesso entusiastici di simili report non ci consentono di cogliere gli specifici bisogni e i punti di forza dei differenti sub-settori, né forniscono indicazioni strategiche per il management culturale. Sarà, dunque, bene indagare la questione da un punto di vista scientifico-teorico, cercando di capire non solo l'origine e le motivazioni che sottendono l'approccio basato sulle ICC, ma anche gli effetti manageriali.

\section{Oltre la retorica della creatività: il framework teorico di riferimento}

\subsection{Dallarmatura culturale all'atmosfera creativa nei processi di sviluppo locale}

Nel corso degli ultimi quindici anni la letteratura internazionale ha riservato un'attenzione crescente all'economia creativa (Howkins, 2001), alle industrie creative (Caves, 2000; Hesmondhalgh, 2007) e alla classe creativa (Florida, 2002), con un progressivo spostamento del dibattito scientifico - ma non solo - dal "culturale" al "creativo". La creatività è così diventata un imperativo economico, ben oltre i tradizionali confini culturali e finanche creativi:

secondo questa retorica, si ritiene che il futuro di uneconomia nazionale competitiva dipenda dalla conoscenza, dalla flessibilità, dalla responsabilità personale e dalle capacità di problem solving dei lavoratori e dei loro manager, i quali, a quanto pare, sono favoriti e incoraggiati da metodi creativi nel business, nell'educazione, nell'industria. Qui, trova spazio un focus particolare sul contributo delle "industrie creative". Questa retorica annette il concetto di creatività al servizio di un programma e di un discorso economici neoliberali (Banaji et al., 2010, p. 70, t.d.a.) ${ }^{6}$.

6 "The future of a competitive national economy is seen to depend, in this rhetoric, on the knowledge, flexibility, personal responsibility and problem solving skills of workers and their managers. These are, apparently, fostered and encouraged by creative methods in business, education and industry. There is a particular focus here on the contribution of the 'creative industries'. This rhetoric annexes the concept of creativity in the service of a neo-liberal economic programme and discourse" (Banaji et al., 2010, p. 70). 
sinergie Vol. 36, N. 105, 2018

In Italia tale retorica è stata supportata anche dalla letteratura economico-manageriale (Sacco, 2010; Santagata, 2009 e 2014; Sedita e Paiola, 2009; Della Lucia, 2014), con un impatto sulle politiche pubbliche nazionali e regionali. Ne forniscono una conferma alcuni recenti progetti di distretto culturale contraddistinti da un passaggio dall"'armatura culturale" all"'atmosfera creativa" (Bertacchini e Santagata, 2012; Cerquetti e Ferrara, 2015; Montella, 2015; Sacco et al., 2015). Tale approccio prevede lapplicazione del modello proposto da Scott (2010) per le grandi citta e le aree metropolitane negli Stati Uniti ai cluster di città di piccole e medie dimensioni: considerando che la localizzazione è un fattore rilevante nei processi creativi (Drake, 2003), ci si aspetta che i cluster culturali funzionino come contesti di fiducia, socializzazione, conoscenza, ispirazione, scambio e innovazione incrementale in un ambiente di produzione caratterizzato da alti livelli di rischio e incertezza (Mommas, 2004, p. 521).

Se si guarda, però, alle indagini condotte dall'OECD sul rapporto tra attività culturali e turismo e sull'impatto della nascita dell'industria creativa su questo rapporto (OECD, 2009 e 2014), si rileva che gli attori principali sono soprattutto le città di maggiori dimensioni, che già da decenni "utilizzano le composite offerte del settore culturale per migliorare il loro posizionamento sui mercati nazionali e internazionali, per accrescere la loro capacità di attrazione sia di flussi di visitatori che di attività economiche e di capitali" (Valentino, 2016, p. 288). Questo dato, sebbene positivo e di incentivo per i contesti urbani, rileva i possibili limiti dell'applicazione di certe politiche nelle aree non metropolitane, spesso marginali e più fragili, che non hanno le potenzialità di innovazione delle grandi città, ma in cui spesso, come in Italia, è capillarmente distribuito il patrimonio culturale.

I tempi non sono ancora maturi per misurare e valutare gli impatti di lungo periodo dei progetti avviati con tale approccio. Tuttavia sono possibili alcune osservazioni di carattere metodologico. Condividendo lapproccio basato sulle risorse, quando si progettano e realizzano distretti culturali, avvalendosi di un'analisi strategica preliminare, occorrerebbe identificare gli asset distintivi che sono alla base del successo locale nel contesto globale (Barney, 1991; Grant, 1991; Montella, 2015). A tal proposito, non sempre l'approccio basato sulle ICC è accompagnato da unanalisi delle risorse uniche ed eccellenti che potrebbero assicurare uno sviluppo di lungo periodo. Più frequentemente, prevale una mera adesione acritica ad un modello cool, probabilmente di successo in altri paesi, senza un'approfondita conoscenza e comprensione dei processi e delle dinamiche capaci di favorire un approccio glocale e sostenibile alleconomia creativa. Il rischio, infatti, è che la creatività - concetto vasto, trasversale, difficile da circoscrivere - possa diventare unetichetta per progetti che non sono realmente legati al territorio. Come afferma Bocci, analizzando i progetti di distretto culturale evoluto avviati in Italia, si è assistito a una

fuga in avanti verso i distretti culturali creativi, senza aver condiviso una visione di sviluppo da parte dei soggetti che progettano lo sviluppo, che non possono che essere le amministrazioni pubbliche, le autonomie locali, i comuni, ovvero in primo luogo chi è il titolare della costruzione di una cornice per una visione di sviluppo sul territorio (Bocci in Feliciati, 2016, p. 106). 
Queste criticità ci impongono unanalisi accurata volta a definire con maggior precisione il significato di "industrie culturali e creative" e a mettere in luce alcune differenze rilevanti al loro interno così come con altri settori. Un auspicabile chiarimento terminologico potrebbe aiutare i policy makers ad evitare l'uso superficiale e l'applicazione fuorviante di vaghe e sovrapponibili etichette. L'obiettivo non è quello di effettuare unanalisi esaustiva del dibattito scientifico sull'argomento, ma piuttosto di evidenziare alcuni aspetti critici: senza puntare a risolvere completamente questioni tassonomiche né tanto meno a chiarire lo stato ontologico ampiamente dibattuto - delle ICC, si vogliono quanto meno mettere in luce gli effetti di certe terminologie sulle politiche culturali.

\subsection{Origini e sviluppo di un dibattito non solo semantico}

Come già suggerito, la mancanza di rigore e l'inconsistente e confusa terminologia attualmente in uso nelle policies sulle ICC derivano dalle debolezze riguardanti la concettualizzazione di cultura e creatività (Galloway e Dunlop, 2007, p. 17). Se quello di cultura è un concetto problematico, allo stesso tempo assolutamente familiare, ma anche complesso e difficile da definire pienamente (O’Brien, 2014, p. 2), ancora più incerto, vago e persino più ampio è il concetto di creatività, così come contraddittorio il suo uso, che può indicare tanto un modo di fare business in qualsiasi settore quanto un preciso settore industriale. Ne consegue che non è facile fornire una definizione chiara e non discutibile non solo delle attività culturali e creative (tab. 1), ma anche del loro valore e della qualità delle relative attività. In aggiunta, considerato che molti ostacoli semantici e politici sono legati all'ancora irrisolta tensione tra cultura ed economia (O'Connor, 2007, p. 7), gli studiosi non sono concordi neanche quando spiegano e discutono il significato di "industria" e "industrie". Come messo in luce da Simon Roodhouse, all'origine del dibattito cè un "contorted and torturous definitional historical discourse" (Roodhouse, 2006, p. 14).

Focalizzando l'attenzione sulle ICC, secondo Throsby, le industrie creative producono beni e servizi creativi, mentre le industrie culturali producono beni e servizi culturali e sono un sottoinsieme del più ampio gruppo delle industrie creative (Throsby, 2010, p. 89). Nel fornire una spiegazione a questa definizione tautologica, nel volume The Economics of Cultural Policy, Throsby definisce i "beni creativi" (creative goods) prodotti che richiedono un livello ragionevolmente significativo di creatività nella loro produzione, senza necessariamente soddisfare altri criteri che permetterebbe loro di essere etichettati come "culturali" (Throsby, 2010, pp. 16-17), e i "beni culturali" (cultural goods) 7 prodotti e servizi che: (1) richiedono un qualche input di creatività umana nella loro produzione; (2) sono veicoli di messaggi simbolici per i consumatori; (3) contengono una qualche forma di proprietà intellettuale; (4) sono soggetti a rational addiction $^{8}$; (5) generano valore culturale in aggiunta a qualsivoglia valore commerciale essi possano avere (Throsby, 2010, p. 16).

7 Da segnalare che la nozione di cultural goods non coincide con l'espressione italiana "beni culturali", corrispondente all'inglese cultural heritage.

8 Rational addiction significa che un incremento nel consumo presente comporterà un incremento nel consumo futuro, in quanto la domanda è cumulativa (Throsby, 2010, p. 16). 


\section{sinergie}

Vol. 36, N. 105, 2018

Sulla stessa linea, O’Connor argomenta che:

le industrie culturali sono quelle attività che hanno a che fare primariamente con beni simbolici - beni il cui primario valore economico deriva dal loro valore culturale. [...] Questa definizione quindi include sia le "classiche" industrie culturali - radio e televisione, cinema, stampa, musica, design, architettura, new media - sia le "arti tradizionali" - arti visive, artigianato, teatro, teatro musicale, concerti e spettacoli, letteratura, musei e gallerie - ovvero tutte quelle attività che hanno i requisiti per essere finanziate pubblicamente come "arte" (O’Connor, 2000, p. 5, t.d.a. $)^{9}$.

La distinzione tra industrie culturali e industrie creative argomentata da Throsby rivela alcune debolezze che è bene mettere in luce. Considerando gli obiettivi di questo lavoro, qui può essere sufficiente prendere in esame tre questioni principali. Innanzitutto, dato che tutte le attività umane necessitano di qualche input creativo e che molte di esse richiedono rilevanti livelli di creatività - indipendentemente dal settore in cui si opera -, ogni industria può essere considerata creativa. In secondo luogo, quello di "messaggio simbolico" o "beni simbolici" - ampiamente discusso nel dibattito scientifico (Martin, 2004, p. 4) - è un concetto ambiguo e volatile, i cui confini sono alquanto incerti: come si chiede qualche studioso, nel contesto attuale è forse possibile escludere una qualche attività produttiva industriale che non abbia un significato simbolico? (Flew, 2002, pp. 1213; Mato, 2009). In aggiunta, il focus sulla natura simbolica, estetica ed artistica di alcuni beni protrae la distinzione tra beni utilitari e non utilitari, che è mistificante in quanto basata su una ristretta definizione di valore economico (Montella, 2016). Infine, se si esclude l'idea che ogni attività umana contiene una qualche forma di proprietà intellettuale, alcune attività connesse al management del patrimonio culturale e il cui output è costituito da servizi culturali dovrebbero essere escluse dal settore culturale, in quanto non possono essere protette da diritti di proprietà intellettuale. La distinzione effettuata da Throsby, dunque, è tutt'altro che chiarificatrice.

Riconoscendo la stretta connessione tra analisi teorico-concettuale e policy making e cercando di mettere da parte il dibattito sulle industrie culturali e/o creative, alcuni studiosi hanno focalizzato l'attenzione sull'emergenza delle ICC nelle policies culturali, in particolare sul passaggio dal "culturale" al "creativo" (Hesmondhalgh e Pratt, 2005), concludendo che le industrie culturali e quelle creative coincidono:

il termine "industrie creative" è una costruzione politica sviluppata per la prima volta dal governo britannico nel 1997 sotto l'amministrazione New Labour. Il termine "industrie culturali" era stato precedentemente utilizzato

9 "The cultural industries are those activities which deal primarily in symbolic goods - goods whose primary economic value is derived from their cultural value. [...] This definition then includes what have been called the 'classical' cultural industries - broadcast media, film, publishing, recorded music, design, architecture, new media - and the 'traditional arts' - visual art, crafts, theatre, music theatre, concerts and performance, literature, museums and galleries - all those activities which have been eligible for public funding as 'art'" (O'Connor, 2000, p. 5). 
dai consigli metropolitani guidati dai laburisti per indicare più o meno le stesse attività. Non cè stata un'esplicita discussione sulle ragioni del cambiamento del termine, né la formulazione di unesauriente definizione da distinguere da quella di "industrie culturali" (o altro termine) (Pratt, 2005, p. 32, t.d.a. $)^{10}$.

Secondo Pratt, l'adozione del nuovo termine ${ }^{11}$ può essere spiegata come il tentativo dei "New" Labour di posizionarsi come politicamente centristi e differenziarsi dalle politiche tendenti a sinistra promosse dagli "Old" Labour. Muovendo dagli stessi assunti, Hewison ha messo in luce come il passaggio dal "culturale" al "creativo" fino alla retorica della creatività suonava non obbligato al passato e suggeriva libertà e autonomia personale (Hewison, 2014, p. 61). Lo zenit di questo processo si è raggiunto con l'invenzione di Creative Britain (Hewison, 2014, p. 39), che lo storico britannico definisce un perfetto esempio dell'ideologia New Labour in continuità con il programma neoliberale messo in atto dai conservatori in vari campi e a vari livelli:

Quando i New Labour iniziarono ad incoraggiare l'individualismo e a lanciare un nuovo spirito imprenditoriale, era necessario utilizzare lo stato per renderlo libero. Per ottenere questo, era necessario dare il via non solo a una riforma istituzionale, ma anche ad un cambiamento culturale. [...] E chi poteva essere contro la creatività? La creatività è positiva e proiettata in avanti - è cool, proprio come i New Labour volevano essere (Hewison, 2014, p. 5, t.d.a. $)^{12}$.

Secondo questa posizione, come meglio argomentato nei paragrafi successivi, pur prendendo le distanze dai Conservatori, i New Labour ne sostenevano il programma economico.

Su questa linea, collocando lo slittamento verso la creatività nel contesto della società globale, Garnham considera l'emergenza del termine "industrie creative" il risultato di cambiamenti industriali, tecnologici e culturali, come lo sviluppo dei media e delle comunicazioni, in particolare delle industrie del copyright e della proprietà intellettuale (Garnham, 2005, pp. 15-16) e, più in generale, dell'affermazione dell'economia dell'informazione o della conoscenza, che è un lascito dell'amministrazione conservatrice (1979-1997). In questa prospettiva, Cunningham (2002)

10 "The term 'creative industries' is a political construct first deployed by the British government in 1997 under a new Labour administration. The term 'cultural industries' had been used previously by Labour-run metropolitan councils to point to more or less the same activities. There was no explicit discussion of why the term changed, nor a statement of a comprehensive definition in distinction to 'cultural industries' (or any other term)" (Pratt, 2005, p. 32).

11 Galloway e Dunlop citano il discorso di John Howkins, all'Inception Session - The Mayor's Commission on the Creative Industries (Londra, 12 dicembre 2002). Sebbene ampiamente sviluppato e diffuso nel Regno Unito, il concetto di "industrie creative" aveva fatto la sua comparsa in Australia già agli inizi degli anni '90 del Novecento (Galloway e Dunlop, 2007, p. 18).

12 "When New Labour set out to encourage individualism and release a new spirit of entrepreneurialism, it had to use the state to set it free. To achieve this, it had to bring about not just institutional reform, but a cultural change. [...] And who could be against creativity? Creativity is positive and forwardlooking - it is cool, just as New Labour wished to be" (Hewison, 2014, p. 5). 
sinergie Vol. 36, N. 105, 2018

argomenta che l'ultima fase del cambiamento tecnologico, includente il World Wide Web e la digitalizzazione, ha sancito il definitivo superamento del vecchio concetto di "industrie culturali" focalizzato sulle arti e sui media commerciali (cinema, radio e televisione, musica) (Galloway e Dunlop, 2007, p. 19).

Infine, Hughson e Inglis hanno salutato le cosiddette "industrie creative" della cultura popolare massmediatica come un tentativo democratico di mettere in discussione le pretese di forme artistiche già legittimate come cultura alta, ovvero pittura, balletto, musica classica e teatro (Hughson e Iglis, 2001, p. 458), sposando gli obiettivi di qualità (ovvero eccellenza) e accesso (Smith, 1998).

Tab. 1: La distinzione tra industrie culturali e creative presente in letteratura

\begin{tabular}{|l|l|}
\hline FONTE & DISTINZIONE TRA INDUSTRIE CULTURALI E CREATIVE \\
\hline $\begin{array}{l}\text { O'Connor, 2000; Throsby, } \\
2010\end{array}$ & $\begin{array}{l}\text { Le industrie creative producono beni e servizi creativi e } \\
\text { richiedono un livello ragionevolmente significativo di creativita } \\
\text { nella loro produzione, senza necessariamente soddisfare } \\
\text { altri criteri che permetterebbe loro di essere etichettate come } \\
\text { "culturali", mentre le industrie culturali producono beni e } \\
\text { servizi culturali e sono un sottoinsieme del più ampio gruppo } \\
\text { delle industrie creative. }\end{array}$ \\
\hline $\begin{array}{l}\text { Hesmondhalgh e Pratt, } \\
\text { Pratt, Hewison, 2014; }\end{array}$ & $\begin{array}{l}\text { Industrie culturali e industrie creative coincidono; il termine } \\
\text { "industrie creative" è una costruzione politica sviluppata } \\
\text { dall'amministrazione New Labour. }\end{array}$ \\
\hline $\begin{array}{l}\text { Cunningham, 2002; } \\
\text { Garnham, 2005; Galloway } \\
\text { e Dunlop, 2007 }\end{array}$ & $\begin{array}{l}\text { Lespressione "industrie creative" è il risultato dell'affermazione } \\
\text { dell'economia dell'informazione o della conoscenza, ovvero } \\
\text { di cambiamenti industriali, tecnologici e culturali (WWW } \\
\text { e digitalizzazione, sviluppo industrie del copyright e della } \\
\text { proprietà intellettuale, etc.). }\end{array}$ \\
\hline Hughson e Iglis, 2001 & $\begin{array}{l}\text { Le industrie creative della cultura popolare massmediatica } \\
\text { sono un tentativo democratico di mettere in discussione le } \\
\text { pretese di forme artistiche già legittimate come cultura alta, } \\
\text { ovvero pittura, balletto, musica classica e teatro. }\end{array}$ \\
\hline
\end{tabular}

Fonte: elaborazione propria

\subsection{Esiti e implicazioni per i settori tradizionali}

Le politiche per le industrie creative hanno stimolato - non solo nel Regno Unito, in cui si è concentrato il dibattito scientifico - un nuovo approccio allo sviluppo locale e alla creazione di cluster culturali basati su: rafforzamento dell'identità, potere di attrazione e posizionamento dei luoghi; promozione di un approccio più imprenditoriale alle arti e alla cultura; incoraggiamento dell'innovazione e della creatività; riuso di vecchi edifici e siti abbandonati; promozione della diversità culturale e della democrazia culturale (Mommaas, 2004). Questorientamento ha inoltre incrementato l'attenzione verso il valore economico della cultura, ma anche verso nuove forme di networking, l'audience development e la partecipazione culturale ${ }^{13}$.

13 Siveda, ad esempio, la controversia tral'approccio basato sulla democratizzazione della cultura e quello basato sulla democrazia culturale: il primo poggia sulla disseminazione top-down delle arti, mentre il secondo sostiene un approccio 
Secondo alcuni studiosi (Galloway e Dunlop, 2007, pp. 28-29), tali approcci, spostando il focus sulla creatività e sulla proprietà intellettuale, hanno progressivamente ignorato le caratteristiche distintive della cultura, contribuendo alla marginalizzazione di attività che sono più legate alla cultura che alla creatività. In particolare, il sotto-settore del patrimonio culturale è stato completamente omesso nel dibattito sulle politiche culturali (Cunningham, 2002). In molti casi, il cultural heritage non è incluso tra le ICC, né considerato accanto agli altri settori culturali e creativi. Una maggiore tensione si è sviluppata, invece, tra le tradizionali forme artistiche e le ICC stricto sensu, per il fatto che in tale dibattito le questioni rilevanti riguardano l'artisticità e il valore artistico. A questa connesse sono le dispute tra produzione di massa e non di massa, tra valore culturale e valore commerciale, tra eccellenza e accesso. Questi dilemmi sono legati anche alla qualità artistica dei prodotti e dei servizi. Talvolta, in tale dibattito si includono i musei, mentre completamente trascurati sono siti archeologici, biblioteche e archivi. Ci sarebbe da chiedersi perché si consideri solo una parte del patrimonio culturale. La ragione è che i musei non vi sono compresi in quanto parte del cultural heritage, ma perché considerati "luoghi dell'arte" - come le gallerie -, sebbene non necessariamente siano musei d'arte.

Per dare al patrimonio culturale una giusta collocazione nelle politiche culturali, si potrebbe allora ripartire dalla distinzione effettuata dal rapporto Kea (2006), rivedendo però i confini del "core arts field", da cui distinguere il patrimonio culturale (tab. 2). Permarrebbero in ogni caso le già citate criticità riguardanti la creatività e i settori creativi.

Pur partendo da diversi presupposti e con qualche differenza, il modello illustrato in questa sede ha punti di contatto con quello proposto da Pietro Antonio Valentino (2012 e 2013), basato sulle rivoluzioni tecnologiche. Tale modello, avvalendosi della struttura a cerchi concentrici utilizzata anche da altri approcci (Throsby, 2008; KEA, 2006), pone nel primo cerchio (subsidized muses) - distinguendoli - beni culturali e arti visive, non in quanto core art fields, ma in quanto attività industriali "più "antiche» realizzate ancora in forme sostanzialmente precapitalistiche" (Valentino, 2013, p. 282), nel secondo cerchio le attività nate con la rivoluzione industriale (industrie culturali) e nel terzo quelle nate con la rivoluzione informatica (industria creativa).

La questione non ha natura esclusivamente semantica o tassonomica, in quanto il framework concettuale e terminologico di riferimento ha un impatto non irrilevante sulle politiche culturali. Per questo si ritiene opportuno adottare un approccio alle politiche culturali che riconosca le differenti caratteristiche dei settori e sub-settori culturali e creativi al fine di: - comprendere e soddisfare gli specifici bisogni delle differenti attività (riproducibili e non riproducibili, soggette e non soggette a copyright, ecc.);

- identificare come promuoverne la cross-fertilization e l'aiuto reciproco;

di tipo bottom-up, attraverso il quale alle persone si insegna ad esplorare il proprio talento creativo fin dai primi anni di vita (Matarasso e Landry, 1999; Hughson e Inglis, 2001, p. 474). 
sinergie

italian journal of management Vol. 36, N. 105, 2018

- valorizzare gli specifici asset locali (patrimonio culturale, attività culturali, ICC, ecc.) in una prospettiva globale;

- promuovere un reale accesso alla cultura, inclusivo sia della comprensione del valore del patrimonio culturale sia dellesplorazione della creatività individuale secondo l'approccio basato sulla democrazia culturale.

Tab. 2: I settori culturali e creativi

\begin{tabular}{|c|c|c|c|}
\hline AMBITI & SETTORI & SUB-SETTORI & CARATTERISTICHE \\
\hline \multirow{2}{*}{$\begin{array}{l}\text { Patrimonio } \\
\text { culturale }\end{array}$} & $\begin{array}{l}\text { Patrimonio culturale } \\
\text { tangibile }\end{array}$ & $\begin{array}{l}\text { Beni e istituti culturali: } \\
\text { musei, "biblioteche, } \\
\text { archivi, "museo diffuso", } \\
\text { paesaggio }\end{array}$ & \multirow{2}{*}{$\begin{array}{l}\text { - Attività non industriali } \\
\text { (servizi) } \\
\text { - Gli output non sono opere } \\
\text { potenzialmente soggette a } \\
\text { copyright }\end{array}$} \\
\hline & $\begin{array}{l}\text { Patrimonio culturale } \\
\text { intangibile }\end{array}$ & $\begin{array}{lr}\begin{array}{l}\text { Tradizioni, } \\
\text { musica, } \\
\text { ecc. }\end{array} & \text { detteratura, } \\
\end{array}$ & \\
\hline \multirow{2}{*}{$\begin{array}{l}\text { Arti } \\
\text { (attività culturali } \\
\text { non riproducibili) }\end{array}$} & Arti visive & \begin{tabular}{|l|} 
Pittura, \\
fotografia, ecc. \\
\end{tabular} & \multirow{2}{*}{$\begin{array}{l}\text { - Attività non industriali } \\
\text { - Gli output sono opere } \\
\text { potenzialmente soggette a } \\
\text { copyright }\end{array}$} \\
\hline & Arti performative & $\begin{array}{l}\text { Teatro, danza, circo, } \\
\text { concerti, festival, ecc. }\end{array}$ & \\
\hline \multirow{5}{*}{$\begin{array}{l}\text { Industrie culturali } \\
\text { (attività culturali } \\
\text { riproducibili) }\end{array}$} & Cinema e video & & \multirow{5}{*}{$\begin{array}{l}\text { - Attività industriali } \\
\text { finalizzate alla riproduzione } \\
\text { di massa } \\
\text { - Gli output sono soggetti a } \\
\text { copyright }\end{array}$} \\
\hline & Radio e televisione & & \\
\hline & Videogame & & \\
\hline & Musica & & \\
\hline & Libri e stampa & & \\
\hline \multirow[b]{3}{*}{$\begin{array}{l}\text { Industrie e attività } \\
\text { creative }\end{array}$} & Design & $\begin{array}{l}\text { Fashion design, graphic } \\
\text { design, interior design, } \\
\text { product design, ecc. }\end{array}$ & \multirow{3}{*}{$\begin{array}{l}\text { - Attività non } \\
\text { necessariamente industriali, } \\
\text { ma che possono essere } \\
\text { prototipizzate } \\
\text { - Anche se gli output non } \\
\text { sono soggetti a copyright, } \\
\text { possono includere altri input } \\
\text { di proprietà intellettuale (es. } \\
\text { marchi registrati, brevetti) } \\
\text { - Luso della creatività è } \\
\text { essenziale per il successo di } \\
\text { questi settori non culturali } \\
\text { (es. abilità creative e risorse } \\
\text { umane provenienti dai } \\
\text { settori delle arti e dal settore } \\
\text { delle industrie culturali) }\end{array}$} \\
\hline & Architettura & & \\
\hline & Pubblicità & & \\
\hline Industrie correlate & ICT, turismo, ecc. & & $\begin{array}{l}\text { - Questa categoria ha confini } \\
\text { vaghi ed è impossibile da } \\
\text { circoscrivere sulla base di } \\
\text { criteri certi; comprende } \\
\text { molti altri settori economici } \\
\text { che dipendono dagli ambiti } \\
\text { precedenti }\end{array}$ \\
\hline
\end{tabular}

Fonte: elaborazione propria da Kea, 2006, p. 3

\section{Il caso di studio}

\subsection{Metodologia e disegno della ricerca}

Sulla base dell'analisi della letteratura sulle industrie culturali e creative e, in particolare, delle criticità riscontrate, la ricerca sul campo si è avvalsa dello studio di caso con una funzione di supporto (supportive role), ovvero 
al fine di testare quanto emerso dalla ricognizione della letteratura. In particolare, ci si è avvalsi di un caso di studio strumentale (instrumental case study) (Stake, 2005, p. 445), come utile strumento esplorativo per spiegare possibili esiti e limiti di politiche che si stanno affermando e diffondendo sempre più ampiamente a livello europeo (Stake, 1983, p. 284).

Partendo da questi presupposti metodologici, nella scelta del caso di studio si è deciso di porre l’attenzione su una realtà dalla quale poter apprendere di più (Stake, 2005, p. 451) e che fosse possibile considerare tipica negli aspetti ritenuti rilevanti per l'argomento oggetto di indagine (Gomm et al., 2000, p. 107). La scelta è così caduta sul Regno Unito, su cui si è focalizzato anche gran parte del dibattito teorico esaminato nei paragrafi precedenti. Il Regno Unito è, infatti, considerato il quartier generale delle politiche e delle strategie basate sulla creatività e sulle ICC, e finanche "the world's creative hub" (Blair, 2007, citato in Holden, 2007), tanto da essere diventato un benchmark per molti altri Paesi (Valentino, 2012, p. 63). Fine della ricerca non è stato quello di ricavare generalizzazioni, ma indicazioni di cui tener conto quando certe politiche vengono esportate in contesti anche molto diversi.

Tenendo conto di quanto previsto dalla letteratura scientifica sullo studio di caso (Yin, 2012 e 2014), l'indagine è stata distinta in due fasi: 1) unanalisi desk, condotta attraverso una review della letteratura comprensiva non solo delle più recenti pubblicazioni sulle politiche culturali del Regno Unito (libri e articoli) ${ }^{14}$, ma anche della letteratura grigia prodotta in tale settore (documenti pubblici, report, green paper, statistiche e altri dati secondari $)^{15}$; 2) unanalisi field di tipo qualitativo, finalizzata a mettere in luce aspetti che non emergono dalle ricerche di tipo quantitativo. Con l'intento di ricavare dati e informazioni utili a una più ampia e completa comprensione del fenomeno e della sua rilevanza ai fini degli specifici obiettivi di questo lavoro, si è scelto di incrociare diversi livelli di analisi (Eisenhardt, 1989, p. 534), quello delle politiche culturali e quello delle imprese operanti nel settore del cultural heritage.

Rispetto ai risultati degli studi prodotti sull'argomento, già ampiamente discussi e diffusi a livello internazionale, si è cercato di comprendere l'impatto delle politiche culturali sulla gestione del patrimonio culturale nel corso degli ultimi venti anni. In particolare, si è scelto di focalizzare l'attenzione sui musei, i quali, secondo la definizione internazionalmente condivisa e aggiornata dall'ICOM nel 2007, sono istituzioni che rivestono un ruolo di primo piano nei processi di tutela e valorizzazione del cultural heritage, in quanto effettuano ricerche sulle "testimonianze materiali e immateriali dell'uomo e del suo ambiente", le acquisiscono, le conservano, le comunicano e specificamente le espongono per scopi di studio, istruzione e diletto.

14 Si segnalano, tra gli altri: Belfiore, 2012; Bell e Oakley, 2015; Cowell, 2007 e 2008; Eisenberg et al., 2006; Garnham, 2005; Gray, 2000; Hesmondhalgh et al., 2015; Hewison, 1987, 1995, 2011 e 2014; Hughson e Inglis 2001; Oakley, 2004; Pendlebury, 2000; Smith, 1998.

15 Tra le fonti prese in esame si vedano in particolare i seguenti rapporti e documenti di policy: CBI, 2014; DCMS, 2011 e 2014; The Warwick Commission, 2015; UK Trade \& Investment, 2014.
Mara Cerquetti Creatività vs patrimonio culturale? The winner takes it all 
sinergie Vol. 36, N. 105, 2018

A tal fine sono state effettuate delle interviste in profondità volte ad indagare il punto di vista di alcuni musei di rilevanza nazionale, presso i quali sono stati realizzati anche sopralluoghi volti a verificare tipologia e qualità dei servizi offerti. Il protocollo di intervista utilizzato è stato elaborato sulla base delle questioni emerse dallesame della letteratura relativamente a: 1) ruolo del patrimonio culturale nelle politiche culturali del Regno Unito; 2) impatto delle politiche promosse di New Labour; 3) rapporto con le politiche europee; 4) impatto del NPM e dell'evidence-based policy, tema su cui il Regno Unito ha fatto da apripista a livello europeo.

Dopo una breve presentazione dell'istituzione (missione, attività e programmi futuri) l'intervista semi-strutturata ha preso in esame gli aspetti dettagliati in tab. 3 .

\section{Tab. 3: Struttura dell'intervista}

\begin{tabular}{|c|c|}
\hline HERITAGE IN THE UK & $\begin{array}{l}\text { - Ruolo del patrimonio culturale nelle politiche culturali del } \\
\text { Regno Unito } \\
\text { - Effetti della commodification del cultural heritage } \\
\text { - Centralità del dibattito sullargomento ed eventuali } \\
\text { questioni emergenti } \\
\text { - Supporto pubblico } \\
\text { - Rapporto tra musei e patrimonio culturale }\end{array}$ \\
\hline NEW LABOUR'S AGENDA & $\begin{array}{l}\text { - Impatto dello slittamento dal "culturale" al "creativo" a cui } \\
\text { si è assistito nel Regno Unito a partire dalla fine degli anni } \\
\text { '90 } \\
\text { - Punti di forza e di debolezza dello slittamento verso la } \\
\text { creatività } \\
\text { - Rapporto tra organizzazioni operanti nel settore dei beni } \\
\text { culturali e ICC } \\
\text { - Sviluppo della cultura imprenditoriale } \\
\text { - Rapporto tra centro e periferia }\end{array}$ \\
\hline EUROPEAN POLICIES & $\begin{array}{l}\text { - Impatto delle politiche europee } \\
\text { - Partecipazione a reti e bandi europei } \\
\text { - Collaborazione con altre istituzioni europee }\end{array}$ \\
\hline $\begin{array}{l}\text { TARGET CULTURE, } \\
\text { PERFORMANCE } \\
\text { MEASUREMENT, } \\
\text { DEMOCRATIZATION OF } \\
\text { CULTURE }\end{array}$ & $\begin{array}{l}\text { - Caratteristiche ed effetti dell'applicazione del NPM al } \\
\text { settore culturale; } \\
\text { - Limiti dell'arm's-length principle } \\
\text { - Punti di forza e di debolezza e spazi di migliorabilità nella } \\
\text { misurazione delle performance (Value for Money, evidence- } \\
\text { based policy e target-driven culture) } \\
\text { - Democratizzazione della cultura }\end{array}$ \\
\hline
\end{tabular}

Fonte: elaborazione propria

Le interviste sono state realizzate tra la fine di marzo e la prima metà di aprile 2016 e hanno coinvolto 5 istituzioni museali londinesi, di diversa tipologia e proprietà ${ }^{16}$ : The Science Museum, The British Museum, The Geffrye Museum of the Home, Royal Museums Greenwich e The Wallace Collection ${ }^{17}$.

16 Ulteriori informazioni sono disponibili nei siti web istituzionali: Science Museum, http://www.sciencemuseum.org.uk; British Museum, http://www. britishmuseum.org/; Geffrye Museum of the Home, http://www.geffryemuseum.org.uk/; Royal Museums Greenwich, http://www.rmg.co.uk/; Wallace Collection, http://www.wallacecollection.org/.

17 Le persone che hanno partecipato alle interviste sono: Helen Jones, Head of Strategy and Planning, Science Museum; Joe Edwards, Department of 
Uno dei primi risultati della ricerca è la conferma della rilevanza del it all tema oggetto di studio, al quale nel Regno Unito negli ultimi anni sono stati dedicati progetti di ricerca, articoli su riviste accademiche e altre pubblicazioni (Gray, 2000; Eisenberg et al., 2006; Hewison, 2014; Bell e Oakley, 2015; Hesmondhalgh et al., 2015).

Analizzando l'argomento nel dettaglio, è opportuno condividere alcune osservazioni preliminari circa il ruolo del patrimonio culturale nelle politiche britanniche, oltre che nella letteratura scientifica. Innanzitutto, a differenza di quanto avviene in Italia e in altri paesi europei ${ }^{18}$, nell'ambito delle politiche e dei finanziamenti culturali, in linea con la distinzione che si riscontra anche in letteratura tra heritage studies e museum studies, nel Regno Unito il patrimonio culturale è un campo distinto dai musei, sebbene non manchino sovrapposizioni e interessi condivisi ${ }^{19}$ (Hesmondhalgh et al., 2015, p. 163).

Pur essendoci strette connessioni con l'industria, nel contesto britannico, le politiche riguardanti il patrimonio culturale hanno avuto una storia differente, oltre che distinta, dalle politiche rivolte alle ICC. A tal proposito, in primo luogo occorre segnalare che il dibattito sulla gestione del patrimonio culturale è stato principalmente riferito alla sua protezione - salvaguardia, conservazione e restauro (Harrison, 2013). Più di recente, il bisogno di protezione del patrimonio culturale ha condotto ad una vera "heritage obsession" (Cowell, 2008), che si è tradotta in un maggior potere di intervento dello stato su beni di proprietà privata. Una conferma di quest'approccio è la creazione dell'Heritage Lottery Fund (HLF). In secondo luogo, il dibattito sul patrimonio culturale nel Regno Unito si è concentrato sulle politiche promosse dai Conservatori per la sua tutela sotto i governi Thatcher e Major, tra il 1979 e il 1997 - sfuggendo persino alla retorica della liberalizzazione. Sebbene nessuno di questi argomenti riesca a spiegare soddisfacentemente tutte le azioni dei governi di questo periodo, Pendlebury prende in esame tre questioni che possono fornire qualche spiegazione di carattere generale circa il successo della tutela sotto i governi conservatori e il grado di consenso senza precedenti riscontrato:

1. the economic commodification of heritage, a partire dalla quale si è iniziato a considerare conservazione e rigenerazione come essenzialmente complementari;

2. the political symbolism of heritage, volto ad utilizzare il passato per obiettivi politici, come un modo per riaffermare l'identità nazionale e "making Britain Great";

3. the dominant ideology thesis, secondo la quale "il patrimonio culturale selezionato per la tutela diviene non semplicemente il riflesso dei

Communication, British Museum; David Dewing, Director, Geffrye Museum of the Home; Christopher Gray, Museum Secretary, Royal Museums Greenwich; Christoph Martin Vogtherr, Director, Wallace Collection.

18 In Italia, ad esempio, i musei sono istituzioni culturali e parte del patrimonio culturale.

19 Per questa ragione, in molti lavori scientifici qui analizzati possiamo trovare riferimenti ai musei - in alcuni casi considerati come un'industria culturale (O'Connor, 2000) -, ma non al patrimonio culturale. 
sinergie Vol. 36, N. 105, 2018

gusti e delle idee delle élite, ma parte di un apparato che assicura il mantenimento del sistema sociale presente" (Pendlebury, 2000, p. 47, t.d.a. ${ }^{20}$.

Il consenso raggiunto da tali politiche in questo periodo ha condotto al progressivo sviluppo dell'heritage industry - innanzitutto la nascita di open air museums e la crescente commodification del cultural heritage (Hewison, 1987 e 1995). Come messo in luce da alcuni critici, ricorrendo ad approcci basati su un forte impatto emotivo, il patrimonio culturale è stato sfruttato per fornire una "versione perfezionata del passato", persino una "storia contraffatta" (bogus history) (Hewison, 1987, p. 10 e p. 144). In particolare, al fine di rendere il patrimonio culturale unattrazione turistica, le country houses e i musei sono diventati teatri di un nostalgico re-enactment del passato come un posto migliore.

Allo stesso tempo, sviluppando l'ideologia della rigenerazione economica, a partire dagli inizi degli anni ' 80 del Novecento altre strategie hanno posto il focus sul ruolo delle industrie culturali per lo sviluppo locale, specialmente per la rigenerazione urbana: le politiche culturali del Greater London Council (GLC) guidato dagli "Old" Labour sono spesso citate come un "momento seminale" (Hesmondhalgh e Pratt, 2005, p. 3), seguite dalle politiche sulle industrie culturali della città di Sheffield e da una grande varietà di progetti di urban regeneration.

Alla fine degli anni '90 i New Labour hanno definitivamente spostato lattenzione verso il settore creativo, comprendente "tutte quelle industrie che hanno la loro origine nella creatività, nell'abilità e nel talento individuali e che hanno un potenziale per la ricchezza e la creazione di lavoro attraverso il generale sfruttamento della proprietà intellettuale" (DCMS, 2001, p. 3, t.d.a. $)^{21}$, così determinando unesplicita innovazione rispetto alle precedenti politiche perseguite sia dai Conservatori sia dagli "Old" Labour. La creatività suggeriva "libertà senza classi e autonomia personale, valori positivi associati con ciò che era inteso in modo crescente come l'economia post-moderna dei segni e dei simboli" (Hewison, 2011, p. 236$, t.d.a. $)^{22}$. Tuttavia, come già argomentato, la creatività è una buzzword, finanche un'idea illusoria (Pratt, 2004, p. 119), non solo difficile da definire, ma presente in molte industrie al di fuori di quelle artistiche. Solo per fornire un esempio della complessità di questargomento, citiamo qui il primo rapporto finalizzato a quantificare il contributo della creatività sulleconomia - il Creative Industries Mapping Document -, in cui il DCMS aveva incluso il "computer software business", che nel 1998, insieme alla pubblicità e al design, copriva almeno la metà del turnover delle industrie creative, mentre il settore culturale filantropico e no profit finanziato dal DCMS costitutiva il 5\% del totale delle industrie creative (Newison, 2014,

20 "The heritage selected for protection becomes not simply a reflection of the tastes and ideas of elites but part of the apparatus that ensure the maintenance of the present social system" (Pendlebury, 2000, p. 47).

21 "those industries that have their origin in individual creativity, skill and talent and which have a potential for wealth and job creation through the general exploitation of intellectual property" (DCMS, 2001, p. 3).

22 "classless freedom and personal autonomy, positive values associated with what was increasingly understood as the post-modern economy of signs and symbols" (Hewison, 20011, p. 236). 
p. 42$)^{23}$. Di conseguenza, l'English Heritage era sempre alla fine della coda per il finanziamento da parte del DCMS (Hewison, 2014, p. 79). Una delle prime azioni che confermano questo nuovo approccio è stato il rebranding del Department of National Heritage, ora Department for Culture, Media and Sport, con un"ideologica" rinuncia al cultural heritage, alla quale hanno fatto seguito la riduzione del supporto economico al patrimonio culturale e la progressiva convergenza economica e tecnologica tra cultura e media (Hewison, 2014, p. 28).

$\mathrm{Da}$ qui in avanti, procedendo sulle orme del brand Cool Britannia ideato dai Conservatori, il patrimonio culturale è diventato parte delle ICC e, in quanto tale, è stato trattato come un'impresa culturale. Secondo Belfiore, la comparsa di Creative Britain ha decretato il successo di unagenda strumentale per le arti e la cultura (Belfiore, 2012, p. 104), ovvero il progressivo ritorno di uno strumentalismo difensivo ed esclusivamente economico, incapace di articolare in modo efficace e significativo argomenti a favore del valore culturale (Belfiore, 2012, p. 107). A giudizio di chi scrive tali osservazioni non sono da leggere nell'ottica di una contrapposizione tra valore culturale e valore economico, ma piuttosto nella prospettiva di una più ampia nozione di valore economico, che non si limiti alla valutazione dei profitti di una singola impresa, assegnando inevitabilmente un ruolo di primo piano alle industrie del copyright, ma che sia in grado di cogliere anche tutte le esternalità, materiali e immateriali, derivanti dal corretto uso del patrimonio culturale (Montella, 2016).

Per quanto riguarda il patrimonio culturale, il focus maggiore sulle ICC è stato accompagnato da una diminuzione dei finanziamenti per il patrimonio culturale, compresi i musei. Sebbene il settore abbia continuato a crescere, la tensione tra accesso ed eccellenza non è stata risolta e gli obiettivi di inclusione sociale e democratizzazione della cultura non sono stati raggiunti completamente. In particolare, unanalisi approfondita rivela due questioni che meriterebbero ulteriori ricerche: (1) da un lato, la persistenza di disuguaglianze sociali; (2) dallaltro, un grande gap tra Londra, dove si concentrano i più importanti musei nazionali e la gran parte dei finanziamenti pubblici e privati, e il resto del paese, dove si fa sentire rilevantemente la diminuzione dei finanziamenti alle autorità locali (Hesmondhalgh et al., 2015).

Si consideri, a titolo di esempio, leffetto dell'ingresso gratuito nei musei, reintrodotto, con un consenso super partes, dai New Labour in tutti gli istituti museali nazionali del Regno Unito nel 2001, e diventato una delle politiche per promuovere l'accesso culturale più visibili e ammirate a livello internazionale (Hesmondhalgh et al., 2015, p. 85; The Warwick Commission, 2015, p. 34) ${ }^{24}$. Anche una misura così ambiziosa, che pure

23 Nel 2010 si è così deciso di escludere tale settore ("business and domestic software design and computer consultancy") dalle stime economiche annuali del DCMS sulle industrie creative (Hewison, 2014, p. 42). Allo stesso tempo, alcuni cambiamenti hanno interessato anche il NESTA (National Endowment for Science, Technology and the Arts), non più finanziato dal DCMS, ma dal Department for Business, Information and Skills (BIS) (Hewison, 2011, p. 239).

24 Sulla linea delle politiche di prezzo introdotte dal Regno Unito, per il caso dell'Italia, si veda il Decreto 27 giugno 2014, n. 94 del Ministro dei Beni e delle it all 
sinergie Vol. 36, N. 105, 2018

ha raggiunto ottimi risultati in termini di aumento dei visitatori, ha fallito lobiettivo di rendere i flagship museums più inclusivi. Analizzandone l'effetto a cinque anni dall'introduzione, Cowell ha segnalato come tra il 2000/2001 e il 2005/2006 le visite da parte delle minoranze etniche siano passate solo dal 3,2\% al 4,9\% del totale, aumento che risulta ancora più scarso se contestualizzato, ad esempio tenendo conto che in una città come Londra - dove si concentrano i principali musei nazionali - le minoranze etniche costituiscono circa il $40 \%$ della popolazione (Cowell, 2007, p. 214). Lanalisi dei dati più recenti riferiti alla partecipazione culturale conferma il gap tra la popolazione bianca e quella appartenente alla categoria BME (Black and Minority Ethnic). Nonostante un incremento complessivo della partecipazione culturale registrato a partire dal 2005/2006, a titolo esemplificativo si rileva che, nei 12 mesi precedenti l'indagine (ottobre 2013/settembre 2014), ha visitato un sito di interesse culturale il 57,7\% della popolazione BME contro il $74,3 \%$ della popolazione bianca, percentuali che scendono rispettivamente al $42,3 \%$ e al $53,1 \%$ se si considera la visita a un museo o una galleria (DCMS, 2014, p. 9 e p. 25).

Di questi limiti occorrerebbe tener conto anche quando certe politiche vengono esportate e riprodotte in paesi con diversa storia e diversa organizzazione del patrimonio culturale, talvolta capillarmente distribuito su tutto il suolo nazionale.

Come confermato dalle interviste ai manager dei musei oggetto di indagine, nel Regno Unito tali politiche sono state finalizzate a promuovere lo sviluppo delle industrie creative e non hanno avuto un impatto rilevante sulle organizzazioni impegnate nella conservazione e valorizzazione del patrimonio culturale. Se alcuni legami con il settore creativo sono stati intensificati, non è stato grazie a particolari incentivi provenienti dalle politiche poste in essere, ma a seguito della necessità di affrontare i cambiamenti globali come la rivoluzione digitale e i bisogni espressi dai nuovi pubblici. Anche le capacità imprenditoriali dei musei non sono il risultato dei recenti tagli finanziari, né la conseguenza degli obiettivi quantitativi di più recente introduzione, ma l'effetto della cultura manageriale introdotta nei musei dalla fine degli anni ' 80 attraverso la sistematica applicazione di target e indicatori di performance. Con qualche eccezione, i soggetti intervistati hanno segnalato anche uno scarso impatto delle politiche europee sul management delle istituzioni culturali e una bassa partecipazione ai progetti europei. Infine, è stato rilevato che nel Regno Unito, diversamente da altri paesi europei, un dibattito sulla gestione del patrimonio culturale è quasi inesistente.

\section{Conclusioni e implicazioni manageriali}

Lesperienza britannica analizzata in questa sede ha avuto un impatto rilevante in Europa, sia ispirando le politiche culturali nazionali (Eisenberg

Attività Culturali e del Turismo (Regolamento recante modifiche al decreto 11 dicembre 1997, n. 507, concernente "Norme per l'istituzione del biglietto di ingresso ai monumenti, musei, gallerie, scavi di antichità, parchi e giardini monumentali dello Stato"), che ha previsto l'ingresso gratuito nei musei statali la prima domenica di ogni mese. 
et al., 2006) sia influenzando l'agenda europea per la cultura e la creatività (European Commission, 2010), in cui il patrimonio culturale è stato inserito sotto l'etichetta "industrie culturali e creative" 25 . Nonostante il continuo successo del settore creativo (CBI, 2014; UK Trade \& Investment, 2014), se l'economia creativa è vista come inevitabile, diviene più difficile comprenderne i punti di debolezza e l'opportunità di una sua revisione (Campbell, 2014, p. 1000) ${ }^{26}$.

Sono stati qui messi in luce i fallimenti nel promuovere l'accesso culturale e nel superare le disuguaglianze sociali, oltre che tra le diverse parti del paese, nonché i limiti nello sviluppo di un'efficace evidence-based policy e nel promuovere la cross-fertilization tra patrimonio culturale e ICC, In particolare, nel contesto "glocale" le diverse performance e i diversi risultati raggiunti dai sub-settori culturali e creativi suggeriscono la necessità di ripensare l'agenda di sviluppo iniziando non dalla promozione delle industrie creative come la strategia vincente adatta ad ogni contesto, ma dalla valorizzazione delle risorse e delle abilità locali (Napolitano e Marino, 2016). Da questo punto di vista, il confronto con nuove prospettive ed approcci può essere un buon punto di partenza. Già nel 2004 Oakley lamentava la sproporzionata influenza del pensiero nordamericano sulle politiche dei New Labour; ad oltre dieci anni di distanza l'Europa può fare valutazioni simili circa l'impronta del pensiero anglosassone sulle politiche culturali nazionali e regionali, oltre che dell'Unione. Come già argomentato (Oakley, 2004, p. 72), se la nozione di industrie creative è problematica, ancora di più lo è l'idea che tale settore possa essere replicato e sviluppato pressoché ovunque, senza alcuna considerazione delle specificità di un luogo. In linea con l'approccio basato sulle risorse già richiamato in questo lavoro, la nascita e lo sviluppo di nuove imprese nel settore culturale ${ }^{27}$ dovrebbero invece valorizzare i place-specific assets dei luoghi, puntando ad una reale cooperazione tra diversi settori e sub-settori industriali e non industriali, non necessariamente riconducibili all'economia creativa, come ad esempio l'enogastronomia, l'artigianato, il made in, il turismo e l'ambiente, se e in quanto capaci di valorizzare risorse idiosincratiche di difficile riproduzione in altri contesti ${ }^{28}$. Parafrasando la canzone di un noto gruppo musicale pop svedese citata nel titolo di questo contributo, cè ancora qualche asso da giocare.

25 Nei successivi documenti di policy europei, le ICC e il patrimonio culturale sono stati considerati settori distinti. Cfr. Council of the European Union, 2010 e 2014.

26 A livello europeo, ad esempio, va registrato che, a fronte della nascita di nuove attività culturali e della riduzione dei costi di produzione, non si è ridotta la dipendenza dal sussidio per le attività artistiche né la necessità della "produzione di massa" per cinema e tv (Valentino, 2012, p. 48).

27 Si veda ad esempio la crescente attenzione allo sviluppo delle start-up nel settore culturale.

28 Focalizzandosi sull'Italia, alcune possibili innovazioni vengono dalla Strategia Nazionale Aree Interne (SNAI, 2013) e dal Piano Strategico di Sviluppo del Turismo 2017-2020 (MiBACT, 2017) recentemente approvato, che mira allo sviluppo di prodotti turistici integrati, facendo dei grandi attrattori culturali come Roma, Firenze, Venezia, Milano delle porte d'accesso al territorio. 


\section{Sinergie}

Vol. 36, N. 105, 2018

\section{Bibliografia}

APPADURAI A. (2015), Banking on Words. The Failure of Language in the Age of Derivative Finance, University of Chicago Press, Chicago.

BANAJI S., BURN A., BUCKINGHAM D. (2010), The rhetorics of creativity: a literature review, CCE Creativity, Culture and Education, Newcastle upon Tyne.

BARNEY J. (1991), "Firm resources and sustained competitive advantage", Journal of Management, n. 17, pp. 99-120.

BELFIORE E. (2012), “'Defensive instrumentalism' and the legacy of New Labour's cultural policies", Cultural Trends, vol. 21, n. 2, pp. 103-111.

BELL D., OAKLEY K. (2015), Cultural policy, Routledge, Abingdon-New York.

BERTACCHINI E., SANTAGATA W. (2012), Atmosfera creativa. Un modello di sviluppo sostenibile per il Piemonte fondato su cultura e creatività, Il Mulino, Bologna.

BUTLER J. (1997), Excitable Speech: A Politics of the Performative, Routledge, New York.

CALVINO I. (1995), "Litaliano, una lingua tra le altre lingue" (1965), in Una pietra sopra, Mondadori, Milano.

CAMMELLI M. (2017), "Il diritto del patrimonio culturale: un'introduzione”, in Barbati C., Cammelli M., Casini L., Piperata G., Sciullo G. (a cura di), Diritto del patrimonio culturale, Il Mulino, Bologna, pp. 11-30.

CAMPBELL P. (2014), "Imaginary Success? - The Contentious Ascendance of Creativity", European Planning Studies, vol. 22, n. 5, pp. 995-1009.

CAUST J. (2003), "Putting the 'art' back into arts policy making: How arts policy has been 'captured' by the economists and the marketers", International Journal of Cultural Policy, vol. 9, n. 1, pp. 51-63.

CAVES R. (2000), Creative Industries: Contracts between Art and Commerce, Harward University Press, Cambridge, MA.

CBI (2014), The creative nation. A growth strategy for the UK's creative industries, Confederation of British Industry, London.

CERQUETTI M. (2014), Marketing museale e creazione di valore: strategie per l'innovazione dei musei italiani, FrancoAngeli, Milano.

CERQUETTI M., FERRARA C. (2015), "Distretti culturali: percorsi evolutivi e azioni di policy a confronto", Il capitale culturale. Studies on the Value of Cultural heritage, Supplementi, n. 3, pp. 137-163.

CHCFE (2015), Cultural heritage Counts for Europe, International Cultural Centre, Krakow.

COMMISSIONE EUROPEA (2010), Le industrie culturali e creative, un potenziale da sfruttare, Libro Verde, Bruxelles, 27.4.2010, disponibile sul sito: http:// eur-lex.europa.eu/legal-content/IT/TXT/?uri=celex\%3A52010DC0183.

COUNCIL OF EUROPE (2005), Council of Europe Framework Convention on the Value of Cultural heritage for Society, CETS No. 199, Faro, 27th Oct. 2005, disponibile sul sito: http://conventions.coe.int/Treaty/EN/Treaties/Html/199. htm; tr. it. http://www.ufficiostudi.beniculturali.it/mibac/multimedia/ UfficioStudi/documents/1362477547947_Convenzione_di_Faro.pdf.

COUNCIL OF THE EUROPEAN UNION (2010), Work Plan for Culture 20112014, Brussels, 18-19 November 2010, available at: http://www.consilium. europa.eu/uedocs/cms_data/docs/pressdata/en/educ/117795.pdf. 
COUNCIL OF THE EUROPEAN UNION (2014), Work Plan for Culture 2015- $\begin{gathered}\text { Mara Cerquetti } \\ \text { Creatività vs }\end{gathered}$ 2018, Brussels, 26 November 2014, available at: http://data.consilium. culturale? The winner takes europa.eu/doc/document/st-16094-2014-init/en/pdf.

COWELL B. (2007), "Measuring the Impact of Free Admission", Cultural Trends, vol. 16, n. 3, pp. 203-224.

COWELL B. (2008), The Heritage Obsession. The Battle for England's Past, The History Press, Stroud.

CUNNINGHAM S.D. (2002), "From cultural to creative industries: Theory, industry, and policy implications", Media International Australia Incorporating Culture and Policy: Quarterly Journal of Media Research and Resources, vol. 102, n. 1, pp. 54-65.

DCMS (2001), Creative Industries Mapping Document, Department for Culture, Media \& Sport, London.

DCMS (2014), Taking Part 2014/15 Quarter 2: Statistical Release, Department for Culture, Media and Sport, December, London, available at: https://www.gov. uk/government/statistics/taking-part-201415-quarter-2-statistical-release.

DELLA LUCIA M. (2014), "La multidimensionalità dello sviluppo locale cultureled. Laboratori territoriali di sperimentazione”, Mercati e Competitività, n. 4, pp. 85-107.

DONATO F. (2013), La crisi sprecata. Per una riforma dei modelli di governance e di management del patrimonio culturale italiano, Aracne, Roma.

DRAKE G. (2003), “This place gives me space': place and creativity in the creative industries", Geoforum, n. 34, pp. 511-524.

E\&Y (2015), Italia Creativa. Primo studio sull'Industria della Cultura e della Creatività in Italia, Ernst \& Young Financial-Business Advisors S.p.A.

EISENBERG C., GERLACH R., HANDKE C., eds. (2006), Cultural Industries: The British Experience in International Perspective, Humboldt University, Berlin, disponibile sul sito: http://edoc.hu-berlin.de/conferences/ culturalindustries/proc/culturalindustries.pdf.

EISENHARDT K.M. (1989), "Building theories from case study research", Academy of Management Review, vol. 14, n. 4, pp. 532-550.

FELICIATI P., a cura di (2016), "La valorizzazione dell'eredità culturale in Italia", Atti del convegno di studi in occasione del $5^{\circ}$ anno della rivista (Macerata, 5-6 novembre 2015), Il capitale culturale. Studies on the Value of Cultural heritage, Supplementi, n. 5, disponibile sul sito: https://riviste.unimc.it/index.php/ cap-cult/issue/view/81.

FLEW T. (2002), "Beyond ad hocery: defining creative industries", paper presented to Cultural sites, Cultural Theory, Cultural Policy, The Second International Conference on Cultural Policy Research (Te Papa, Wellington, New Zeland, 23-26 January 2002), available at: http://eprints.qut.edu.au/256/1/Flew_ beyond.pdf.

FLORIDA R. (2002), The Rise of the Creative Class: And How It's Transforming Work, Leisure, Community and Everyday Life, Basic Books, New York.

GALLOWAY S., DUNLOP S. (2007), "A critique of definitions of the cultural and creative industries in public policy", International Journal of Cultural Policy, vol. 13, n. 1, pp. 17-31.

GARNHAM N. (2005), "From cultural to creative industries", International Journal of Cultural Policy, vol. 11, n. 1, pp. 15-29. 
GOMM R., HAMMERSLEY M., FOSTER P. (2000), “Case study and generalization”, in Case study method, Sage Publications, Thousand Oaks, CA, pp. 98-115.

GRANT R.M. (1991), “The Resource-based Theory of Competitive Advantage: Implications for Strategy Formulation", California Management Review, n. 33, pp. 114-135.

GRAY C. (2000), The Politics of the Arts in Britain, Palgrave Macmillan, London. HARRISON R. (2013), Heritage. Critical Approaches, Routledge, Abingdon-New York. HESMONDHALGH D. (2007), The Cultural Industries, Sage, London.

HESMONDHALGH D., OAKLEY K., LEE D. (2015), Culture, Economy and Politics. The Case of New Labour, Palgrave Macmillan, London.

HESMONDHALGH D., PRATT A.C. (2005), "Cultural industries and cultural policy”, International Journal of Cultural Policy, vol. 11, n. 1, pp. 1-14.

HEWISON R. (1987), The Heritage industry. Britain in a climate of decline, A Methun Paperback, York.

HEWISON R. (1995), Culture \& Consensus. England, art and politics since 1940, Methuen, London.

HEWISON R. (2011), “Creative Britain: myth or monument?", Cultural Trends, vol. 20, n. 3, pp. 235-242.

HEWISON R. (2014), Cultural Capital: The Rise and Fall of Creative Britain, Verso, London-New York.

HOLDEN J. (2007), Publicly Funded Culture and the Creative Industries, Arts Council, London.

HOWKINS J. (2001), The Creative Economy: How People Make Money from Ideas, Penguin, London.

HUGHSON J., INGLIS D. (2001), “Creative industries' and the arts in Britain: Towards a 'third way' in cultural policy?", International Journal of Cultural Policy, n. 7, pp. 457-478.

KEA EUROPEAN AFFAIRS (2006), Economy of Culture in Europe, European Commission, Directorate-General for Education and Culture, Brussels.

MARTIN C. (2004), "Defining Culture and Communication for the Purpose of National and International Statistics Program", paper presented to the $3^{\text {rd }}$ International Conference on Cultural Policy Research (HEC Montréal, Montreal, Canada, 25-28 August 2004), available at: http://neumann.hec. ca/iccpr/PDF_Texts/Martin_Claude.pdf.

MATARASSO F., LANDRY C. (1999), Balancing act: twenty-one strategic dilemmas in cultural policy, Council of Europe, Strasbourg.

MATO D. (2009), "All industries are cultural. A critique of the idea of 'cultural industries' and new possibilities for research", Cultural Studies, vol. 23, n. 1, pp. 70-87.

MiBACT (2017), PST 2017-2020. Italia Paese per Viaggiatori, Piano Strategico di Sviluppo del Turismo, Invitalia, Roma, disponibile sul sito: http://www.pst. beniculturali.it/wp-content/uploads/2017/03/PST_2017_IT_final.pdf.

MOMMAAS H. (2004), "Cultural clusters and the post-industrial city: Towards the remapping of urban cultural policy", Urban Studies, n. 41, pp. 507-532.

MONTELLA M. (2015), "Quale distretto culturale - fra accademia e fattività", $I l$ capitale culturale. Studies on the Value of Cultural heritage, Supplementi, n. 3, pp. 11-42.

MONTELLA M. (ed.) (2016), Economia e gestione delleredità culturale. Dizionario metodico essenziale, Wolters Kluwer-CEDAM, Alphen aan den Rijn-Milano. 
NAPOLITANO M.R., MARINO V. (2016), Cultural heritage e Made in Italy. Casi ed esperienze di marketing internazionale, Editoriale Scientific, Napoli.

OAKLEY K. (2004), "Not so cool Britannia: The role of the creative industries in economic development", International Journal of Cultural Studies, vol. 7, n. 1, pp. 67-77.

O'BRIEN D. (2014), Cultural policy. Management, value and modernity in the creative industries, Routledge, Abingdon-New York.

O'CONNOR J. (2000), "The definition of the 'Cultural Industries", The European Journal of Arts Education, vol. 2, n. 3, pp. 15-27, available at: http://eprints. qut.edu.au/43877/2/43877.pdf.

O'CONNOR J. (2007), The cultural and creative industries: a review of the literature, Arts Council England, Creative Partnerships, London.

OECD (2009), The impact of Culture on Tourism, OECD Publishing, Paris, available at: http://www.mlit.go.jp/kankocho/naratourismstatisticsweek/statistical/ pdf/2009_The_Impact.pdf.

OECD (2014), Tourism and the Creative Economy, OECD Studies on Tourism, OECD Publishing, Paris, available at: http://www.mlit.go.jp/kankocho/ naratourismstatisticsweek/statistical/pdf/2014_Tourism_and_the_ creative.pdf.

PENDLEBURY J. (2000), "Conservation, Conservatives and Consensus: The Success of Conservation under the Thatcher and Major Governments, 1979-1997", Planning Theory and Practice, vol. 1, n. 1, pp. 31-52.

PRATT A.C. (2004), "The cultural economy. A call for spatialized 'production of culture' perspectives", International Journal of Cultural Studies, vol. 7, n. 1, pp. 117-128.

PRATT A.C. (2005), "Cultural industries and public policy: an oxymoron?", International Journal of Cultural Policy, vol. 11, n. 1, pp. 31-44.

ROODHOUSE S. (2006), “The Creative Industries: Definitions, Quantification and Practice”, in Eisenberg C., Gerlach R., Handke C., eds., Cultural Industries: The British Experience in International Perspective, Humboldt University, Berlin, pp. 13-31, available at: http://edoc.hu-berlin.de/conferences/ culturalindustries/proc/culturalindustries.pdf.

SACCO P.L. (2010), "Cultura e sviluppo locale: il distretto culturale evoluto", Sinergie, n. 82, pp. 115-119.

SACCO P.L., FERILLI G., TAVANO BLESSI G. (2015), Cultura e sviluppo locale. Verso il distretto culturale evoluto, Il Mulino, Bologna.

SANTAGATA W. (2009), Libro bianco sulla creatività. Per un modello italiano di sviluppo, Università Bocconi, Milano.

SANTAGATA W. (2014), Il governo della cultura. Promuovere sviluppo e qualità sociale, Il Mulino, Bologna.

SCOTT A.J. (2010), "Cultural Economy and the Creative Field of the City", Geografiska Annaler: Series B, Human Geography, vol. 92, n. 2, pp. 115-130.

SEDITA S.R., PAIOLA M., a cura di (2009), Il management della creatività. Reti, comunità e territori, Carocci, Roma.

SMITH C. (1998), Creative Britain, Faber and Faber, London.

SNAI (2013), Strategia nazionale per le Aree interne: definizione, obiettivi, strumenti e governance (Accordo di Partenariato 2014-2020), documento tecnico collegato alla bozza di Accordo di Partenariato trasmessa alla CE il 9 dicembre 2013. it all 
STAKE R.E. (1983), “The case study method in social inquiry”, in Evaluation models, Springer Netherlands, pp. 279-286.

STAKE R.E. (2005). "Qualitative case studies", in Denzin N.K., Lincoln Y.S., eds., The Sage Handbook of Qualitative Research, ${ }^{\text {rd }}$ Edition, Sage Publications, Thousand Oaks, CA, pp. 443-466.

SYMBOLA, UNIONCAMERE (2015), Io sono cultura. L'Italia della qualità e della bellezza sfida la crisi, Quaderni di Symbola, Symbola, Unioncamere, Roma.

SYMBOLA, UNIONCAMERE (2016), Io sono cultura. L'Italia della qualità e della bellezza sfida la crisi, Quaderni di Symbola, Symbola, Unioncamere, Roma.

THE WARWICK COMMISSION (2015), Enriching Britain: Culture, Creativity and Growth. The 2015 Report by the Warwick Commission on the Future of Cultural Value, University of Warwick, Warwick.

THROSBY (2008), "Modelling the cultural industries", International Journal of Cultural Policy, vol. 14, n. 3, pp. 217-232.

THROSBY D. (2010), The Economics of Cultural Policy, Cambridge University Press, Cambridge.

UK TRADE \& INVESTMENT (2014), UK Creative Industries - International Strategy. Driving global growth for the UK creative industries, UK Trade \& Investment, London.

VALENTINO P.A., a cura di (2012), Larte di produrre arte: imprese culturali a lavoro, Marsilio, Venezia.

VALENTINO P.A. (2013), "L'impresa culturale e creativa: verso una definizione condivisa", Economia della cultura, n. 3, pp. 273-288.

VALENTINO P.A. (2016), I mutamenti nelleconomia della cultura, in Impresa Cultura. Creatività, Partecipazione, Competitività, $12^{\circ}$ Rapporto Annuale Federculture 2016, Gangemi, Roma, pp. 281-293.

YIN R.K. (2012), Applications of Case Study Research, Third Edition, Sage publications, Thousand Oaks, CA.

YIN R.K. (2014), Case Study Research: Design and Methods, Fifth Edition, Sage publications, Thousand Oaks, CA.

\section{Academic or professional position and contacts}

Mara Cerquetti

Researcher in Management

University of Macerata - Italy

e-mail: mara.cerquetti@unimc.it

sinergie italian journal of management ISSN 0393-5108 DOI $10.7433 / \mathrm{s} 105.2018 .06$ ONDAZIONE CUEIM 